Published in final edited form as:

Nanomedicine. 2010 February ; 6(1): 64-69. doi:10.1016/j.nano.2009.04.002.

\title{
Multifunctional Magnetic Nanoparticles for Targeted Delivery
}

\author{
Arun Kumar, $\mathrm{PhD}^{1}$, Prasanna K. Jena, $\mathrm{PhD}^{1}$, Sumita Behera, $\mathrm{PhD}^{2}$, Richard F. Lockey, \\ MD $^{1,4}$, Subhra Mohapatra, $\mathbf{P h D}^{3,4}$, and Shyam Mohapatra, $\mathbf{P h D}^{1,4}$ \\ ${ }^{1}$ Division of Allergy and Immunology, Department of Internal Medicine, University of South \\ Florida, Tampa, Florida \\ ${ }^{2}$ TransGenex Nanobiotech Inc., Tampa, Florida \\ ${ }^{3}$ Divison of Endocrinology, Department of Internal Medicine, University of South Florida, Tampa, \\ Florida \\ ${ }^{4}$ VA Hospital Research, James A. Haley Hospital, Tampa, Florida
}

\begin{abstract}
A major problem associated with therapy is the inability to deliver pharmaceuticals to a specific site of the body without causing nonspecific toxicity. Development of magnetic nanoparticles and techniques for their safe transport and concentration in specific sites in the body would constitute a powerful tool for gene/drug therapy in vivo. Furthermore, drug delivery in vitro could improve further if the drugs were modified with antibodies, proteins or ligands. For in vivo experiments, magnetic nanoparticles were conjugated with plasmid DNA expressing GFP and then coated with chitosan. These particles were injected into mice through tail vein and directed to heart and kidney by means of external magnets of 25 gauss or $2 \mathrm{kA}-\mathrm{kA} / \mathrm{m}$. These particles were concentrated in the lungs, heart, and kidney of mice and the expression of GFP in these sites were monitored. The expression of GFP in specific locations was visualized by whole-body fluorescent imaging and the concentration of these particles in the designated body locations was confirmed by transmission electron microscopy. In another model system, we used atrial natriuretic peptide (ANP) and Carcino Embryonic Antigen (CEA) antibodies coupled to the chitosan coated magnetic nanoparticles to target cells in vitro. The present work demonstrates that a simple external magnetic field is all that is necessary to target a drug to a specific site inside the body without the need to functionalize the nanoparticles. However, the option to use magnetic targeting with external magnets on functionalized nanoparticles could prove as a more efficient means of drug delivery.
\end{abstract}

\section{Keywords}

magnetic nanoparticles; gene therapy; iron oxide; localization; chitosan

\footnotetext{
(C) 2009 Elsevier Inc. All rights reserved.

*Corresponding author: Division of Allergy and Immunology and Department of Internal Medicine, University of South Florida College of Medicine and VA Hospital, 12908 USF Health Dr., Tampa, FL 33612, Ph: 813-974 8568, Fax: 813-974-8575, smohapat@health.usf.edu .

Publisher's Disclaimer: This is a PDF file of an unedited manuscript that has been accepted for publication. As a service to our customers we are providing this early version of the manuscript. The manuscript will undergo copyediting, typesetting, and review of the resulting proof before it is published in its final citable form. Please note that during the production process errors may be discovered which could affect the content, and all legal disclaimers that apply to the journal pertain.
} 


\section{Introduction}

Magnetic drug targeting, i.e. the targeting of a drug immobilized on a magnetic material under the action of an external magnetic field, constitutes an important drug delivery scheme [1]. The use of superparamagnetic iron oxide nanoparticles (SPIONs) for the delivery of therapeutic molecules holds great potential for clinical applications, as it could provide substantial improvement over current techniques for drug delivery and gene transfection. The magnetic field would pull the SPIONs to the target organ or tissue, where the biodegradable vehicles would subsequently break down and release drugs, DNA plasmids or bioactive molecules into surrounding tissues. This would solve many of the problems associated with current drug therapy methods, such as the nonspecific concentration of drugs in healthy sites causing toxicity and other adverse side effects in the bystander cells.

Ideally, the drug, peptide, or gene could be attached to strongly magnetic particles and wrapped with a material that could evade the body's defense system and protect the drug from premature degradation. Iron oxides, such as magnetite, are the most commonly used compounds for particle synthesis, because the low oxidizing nature of ferromagnetic materials maintains a stable magnetic response.

Magnetic nanoparticles are physiologically inert, with no measurable LD50 [2,3], but when they enter the bloodstream, they are rapidly coated with plasma proteins in a process known as opsonization [4]. The opsonized particles may be recognized by the reticuloendothelial system (RES) and macrophages may remove them by phagocytosis. Specific modification to the surface of the nanoparticles could increase the circulation time by making the particles invisible to the RES. RES evasion could be accomplished by coating the particles with a layer of hydrophilic materials such as chitosan [5].

The nanoparticle target specificity could also be further enhanced if the drug-loaded nanoparticles were conjugated with antibodies, lectins, proteins, hormones, charged molecules, or low molecular weight ligands capable of recognizing and binding to the specific target site [6]. These particles could target specific cells, such as tumor cells, while protecting the drugs from premature inactivation during transport.

In the current work, we combined the transfecting and drug/DNA/peptide protecting capability of chitosan with a super paramagnetic particle $\left(\mathrm{Fe}_{2} \mathrm{O}_{3}\right)$ to synthesize hydrophobic magnetic nanoparticles. Chitosan coated magnetic $\mathrm{Fe}_{2} \mathrm{O}_{3}$ particles combined with plasmid DNA expressing green fluorescent protein (GFP) were injected into mice and directed to the lungs, heart, and kidney in vivo by means of external magnets of 25 gauss or $2 \mathrm{kA}-\mathrm{kA} / \mathrm{m}$. The expression of GFP in these sites was visualized by whole-body fluorescent imaging. We have also made magnetic nanoparticles conjugated with ANP peptide or CEA antibodies to transfect cells in vitro.

In this report, we demonstrate that a simple external magnetic field is all that is necessary to target a drug to a specific site inside the body. There is no need to functionalize the nanoparticles with peptides or antibodies to achieve drug localization. However, combining magnetic targeting with functionalized nanoparticles could further enhance the target specificity.

\section{Methods}

HEK293, A549 and WI-38 cells were obtained from ATCC and maintained in Dulbecco's modified Eagle's medium (Gibco) supplemented with $10 \%$ fetal bovine serum (v/v) and $1 \%$ Pen/Strep under a humidified atmosphere of $5 \%$ carbon dioxide and $95 \%$ air at $37^{\circ} \mathrm{C}$. A GFP-expressing plasmid was obtained from Stratagene, amplified in DH5a E. coli grown in 
LB (Invitrogen) and purified using a MaxiPrep kit (Qiagen, Valencia, CA). Chitosan was obtained from Vanson (Redmond, WA). Mice were purchased from Jackson Labs and housed in a pathogen-free environment. Magnetic nanoparticles were characterized by TEM performed using a Hitachi Model 7280. FTIR spectra were obtained using a Perkin Elmer instrument. The PAVERA FITC labeling kit was used to label all the nanocomplexes with fluorescein. Iron (II) chloride, iron (III) chloride, and $\mathrm{NH}_{4} \mathrm{OH}(25 \%)$ were obtained from Aldrich (St. Louis, MO USA), CEA antibodies were from Invitrogen, and ANP peptide was procured from Sigma (St. Louis, MO). In all preparations, Nanopure water (Millipore) of resistance $17.8 \mathrm{M} \Omega$ was used.

\section{Synthesis of $\mathrm{Fe}_{2} \mathrm{O}_{3}$ nanoparticles}

$\mathrm{Fe}_{2} \mathrm{O}_{3}$ nanoparticles were synthesized in aqueous medium without surfactants. Colloidal magnetite suspensions were directly oxidized by aeration to form $\mathrm{Fe}_{2} \mathrm{O}_{3}$ nanoparticles. To synthesize homogeneous nanoparticles and compositions, the reaction was done with vigorous stirring in basic aqueous solutions with a molar ratio of Fe (II) / Fe (III) of 1:2. After formation, the nanoparticles were washed several times with DI water to remove unreacted components. The $\mathrm{Fe}_{2} \mathrm{O}_{3}$ nanoparticles have an average diameter of $60-70 \mathrm{~nm}$ and a narrow size distribution [7].

\section{Chemical Reaction-}

$\mathrm{FeCl}_{2}+\mathrm{FeCl}_{3} \longrightarrow \mathrm{Fe}_{3} \mathrm{O}_{4} \longrightarrow \mathrm{Fe}_{2} \mathrm{O}_{3}$

\section{Coupling magnetic nanoparticles to pDNA}

A $0.2 \mathrm{M}$ suspension of $\mathrm{Fe}_{2} \mathrm{O}_{3}$ was made in sterile DI water. The suspension was deoxygenated with $\mathrm{N}_{2}$ gas for 2-3 min then added to a solution of pEGFP DNA at a ratio of 1:1 (v/v). After 20 min incubation at $55^{\circ} \mathrm{C}$, the pEGFP DNA- $\mathrm{Fe}_{2} \mathrm{O}_{3}$ complex was mixed with water-soluble chitosan at a DNA to chitosan ratio of 1:5 (w/w) The pDNA- $\mathrm{Fe}_{2} \mathrm{O}_{3}$ chitosan complex was incubated at $55{ }^{\circ} \mathrm{C}$ for $20 \mathrm{~min}$ with intermittent shaking and separated from uncomplexed reagents by means of a magnet. The complex was resuspended in sterile water and $10 \mathrm{ug}$ of nanoparticle/DNA complex was injected into the tail veins of each of four mice. These mice were separated into two groups of two mice each. In one group, a circular magnet of 25 gauss or $2 \mathrm{kA}-\mathrm{kA} / \mathrm{m}$, wrapped in cheese cloth, was tied between the front legs of each mouse for about $6 \mathrm{~h}$ to target the heart. In the other group, the magnets were tied between the back legs to target the kidneys. Mice were sacrificed after $12 \mathrm{~h}$ and subjected to bronchoalveolar lavage to detect EGFP-positive cells in the BAL. Control mice were also given the chitosan magnetic nanoparticles coupled with EGFP, but was not exposed to an external magnet. Hearts and kidneys were collected, fixed, sectioned and examined by fluorescent microscopy for EGFP-positive (green fluorescent) cells.

\section{Coupling magnetic nanoparticles to ANP peptide}

ANP peptide- $\mathrm{Fe}_{2} \mathrm{O}_{3}$-chitosan complexes were synthesized using the stock solution of $\mathrm{Fe}_{2} \mathrm{O}_{3}$ nanoparticles. The stock solution of $2.50 \mathrm{mg} / \mathrm{mL}$ was prepared by dissolving $\mathrm{Fe}_{2} \mathrm{O}_{3}$ in DI water, and magnetic iron oxide $\left(\mathrm{Fe}_{3} \mathrm{O}_{4}\right)$ and chitosan nanoparticles were dispersed in DI water prior to modification with peptide. Chitosan was first carboxymethylated and then covalently bound on the surface of $\mathrm{Fe}_{3} \mathrm{O}_{4}$ nanoparticles via carbodiimide activation. This solution was washed several times with DI water prior to use. Chitosan-coated $\mathrm{Fe}_{2} \mathrm{O}_{3}$ nanoparticles were resuspended in water and mixed with ANP peptide at a ratio of 1:1 (w/ w). Gluteradehyde was then added to a final concentration of $0.2 \%$. The mixture was stirred for $4-5 \mathrm{~h}$ at $40{ }^{\circ} \mathrm{C}$ to couple the $\mathrm{Fe}_{2} \mathrm{O}_{3}$-coated nanoparticles to the peptide. The coupled 
particles were washed twice with DI water, air dried and left in a vacuum oven for $48 \mathrm{~h}$ to remove all traces of water. The dried film was resuspended in DI water with agitation and the solution was filtered through a cellulose membrane.

\section{Coupling magnetic nanoparticles to CEA antibody}

A solution of CEA antibodies in DI water was mixed with $0.5 \mathrm{~mL}$ of carbodiimide solution and added to a colloidal suspension of magnetic nanoparticles. The solution was left to stand for 15 min to allow hydrolysis of the surface groups and formation of vitreophilic nanoparticles. The functionalized suspension was stirred vigorously and allowed to stand for at least $24 \mathrm{~h}$ to produce $\mathrm{CEAAb}-\mathrm{Fe}_{2} \mathrm{O}_{3}$ nanoparticles.

\section{Characterization of modified magnetic nanoparticles}

Homogenous iron nanoparticles were made with Fe (II): Fe (III) at a molar ration of $1: 2$ by reactions under basic aqueous conditions without surfactants. The aqueous suspension was precipitated and the precipitates were washed with DI water. Figure 1 shows a transmission electron microscopy (TEM) picture of the $\mathrm{Fe}_{2} \mathrm{O}_{3}$ particles. The average diameter of the LAMPs was determined by TEM and varies from 60 to $70 \mathrm{~nm}$. The coupling of peptides was confirmed by TEM and the coupling of antibodies was confirmed by Fourier transform infrared spectroscopy (FTIR) (Figure $2 \& 3$ ).

\section{Targeting of cancer cells with multifunctional magnetic nanoparticles}

To test the specificity of antibody-based targeting, magnetic nanoparticles were coupled to an antibody to carcino embryogenic antigen (CEA). Human A549 lung adenocarcinoma and human lung diploid cell line, WI-38 (normal human fibroblast) cells were seeded in 8chamber well slides $\left(10^{5}\right.$ cells/well) and transfected with magnetic nanoparticles containing CEA antibody-FITC coupled to either chemiluminescent reagent BPI-101 or BPI-108. After $48 \mathrm{~h}$, the cells were washed with phosphate-buffered saline (PBS), fixed with $4 \%$ paraformaldehyde for $20 \mathrm{~min}$ and washed with PBS twice. The slides were mounted with the nuclear stain, diaminopimelimidate (DAPI), examined under the fluorescence microscope and photographed. HEK293 and HEK293-hcg cells were transfected with nanoparticles containing $\mathrm{Fe}_{2} \mathrm{O}_{3}$ with ANP-conjugated-FITC. After $48 \mathrm{~h}$, the cells were washed with PBS, fixed with $4 \%$ paraformaldehyde for 20 min, washed with PBS twice, mounted with DAPI, and examined by fluorescence microscopy.

\section{Results}

\section{Localization of drug delivery using eGFP conjugated magnetic nanoparticles at specific site with external magnetic influence}

It was determined by agarose gel electrophoresis and ethidium bromide staining that about $80 \%$ of the complexes constituted pDNA-Fe-chitosan (data not shown). This factor was taken into consideration for calculating the amount of complexed DNA injected into mice. The size of the pDNA-Fe complex was 60-70 nm, while the size of the pDNA-Fe-chitosan complex was found to be $>100 \mathrm{~nm}$ (Figure 4A). Figure 4B shows GFP expression in BAL cells from a mouse with the external magnet tied near the heart and a control mouse without a magnet. Sections of the heart tissue from the mouse with the magnet show extensive expression of GFP in comparison with the control mouse without magnet (Figure 4C).

\section{Cell targeting with multifunctional magnetic nanoparticles}

Magnetic nanoparticles linked to CEA antibodies and ANP peptides were transfected into human cancer cell lines and control cell lines. The ANP peptide bound magnetic particles were transfected into the HEK293hcg cell line; while the HEK293 cell line was used as a 
control. ANP peptides bind to over expressed ANP receptors on HEK293hcg cells and initiate endocytosis, but not in the HEK293 cells (Figure 5). The CEA antibody bound magnetic particles were transfected in to the A549 cell lines; while the WI-38 cells lines were used as the control. CEA antibodies bind specifically to CEA proteins on the surface of A549 cancer cells and induce endocytosis of the nanoparticle peptide complex across the plasma membrane, but not in the WI-38 cells (Figure 6).

\section{Discussion}

When a foreign particle enters the bloodstream it is opsonized and removed by the body's RES system. Particles that have a highly hydrophobic surface are rapidly opsonized, while those with a more hydrophilic surface resist ionizations and are cleared slowly [8]. RESevading particles have been synthesized by stabilizing the particles with a layer of hydrophilic material [9]. Chitosan is a hydrophilic aminosaccharide polymer that can be used to coat the iron particles and increase their circulation time in the bloodstream by evading the RES. Simberg et al. increased the half-life of iron nanoparticles 5-fold by depleting RES macrophages in the liver with liposomal clodronate [10]. In an iron nanoparticle-based diagnostic experiment, they also blocked RES uptake by pretreating with a Ni-liposome decoy.

In the in vivo study, we did not perform pretreatment to block uptake; since we studied the accumulation of chitosan-Fe-pDNA nanoparticles in the heart and kidney, which are nonRES tissues. Furthermore, the time these particles spent in the circulation was considerably reduced by rapidly dragging them to the heart and kidney with an external magnet. Chitosan coating of nanoparticles was used to improve GFP expression by combining the DNA protection of chitosan with its DNA transfecting characteristics.

Magnetic $\mathrm{Fe}_{2} \mathrm{O}_{3}$ nanoparticles were used to facilitate localization of the pDNA to the heart and kidney under the influence of an external magnet. It is clear that the magnetic field applied for $6 \mathrm{~h}$, effectively directed and concentrated the pDNA-Fe-chitosan complexes to the target sites of the heart and trachea. The size and magnetic properties of the nanoparticles were optimized to reduce RES uptake and to increase the circulation time in the organism. The field strength of the magnet should also be high. This will provide maximum concentration of the nanoparticles in the target area, because as the circulation time increases, there is more possibility of loss of nanoparticles [2]. Our investigation has shown that there will be about 30 percent loss in the nanoparticles during the course of dragging them at specific site (data not shown). Using different types of iron nanoparticles and magnets of various field strengths, a number of investigators have successfully concentrated particles in target tissues [2]. In the absence of an external magnetic field, the super-paramagnetic particles lose their magnetism and do not cause aggregation [11,12]. The present work demonstrates that a simple external magnetic field is all that is necessary to target a drug to a specific site inside the body without the need to functionalize the nanoparticles.

However, functionalized nanoparticles containing antibodies or peptides could also be attached to magnetic particles and directed to specific body sites. Coupling magnetic nanoparticles with antibodies or targeting molecules and dragging them to specific site using external influence could be a more effective tool to deliver the drug more effectively.

There have been many uses of magnetic particle manipulation in the human body; however, a single system has yet to emerge that combines those techniques into a simple tool for a wide range of applications used by the medical community. Magnetically targeted drug 
delivery is a viable technology, but it is still in its infancy and there is much work to be completed before an effective clinical solution emerges.

\section{Acknowledgments}

This work was supported by national Institutes of Health (NIH) (5RO1HL71101-01A2), the James A. Haley Veteran's Hospital and the I-4 Corridor support and the Mabel and Ellsworth Simmons endowments to SSM.

\section{References}

1. Berry CC, Curtis ASG. Functionalization of magnetic nanoparticles for application in biomedicine. J. Phys. D: Appl. Phys. 2003; 36:R198-R206.

2. Lubbe AS, Alexiou C, Bergemann C. Clinical applications of magnetic drug targeting. J. Surg. Res. 2001; 95:200-206. [PubMed: 11162046]

3. Babincova M, Sourivong P, Leszczynska D, Babinec P. Blood-specific whole-body electromagnetic hyperthermia. Med. Hyptoth. 2000; 55:459-460.

4. Davis SS. Biomedical applications of nanotechnology-implications for drug targeting and gene therapy. Trends Biotechnol. 1997; 15:217-224. [PubMed: 9183864]

5. Lacava LM, et al. Magnetic resonance of a dextran-coated magnetic fluid intravenously administered in mice. Biophys. J. 2001; 80:2483-2486. [PubMed: 11325747]

6. Sudimack J, Lee RJ. Targeted drug delivery via folate receptor. Adv. Drug Del. Rev. 2000; 41:14712.

7. Kumar A, Fal-Miyar V, García JA, Cerdeira A, Mohapatra S, Srikanth H, Kurlyandskaya GV. Magnetoimpedance biosensor for Fe3O4 nanoparticles intracellular uptake evaluation. Applied Physics Letters. 2007; 91:143902.

8. Katz E, Baron R, Willner I. Magnetoswitchable electrochemistry gated by alkyl-chainfunctionalized magnetic nanoparticles: Control of diffusional and surfaceconfined electrochemical processes. J. American Chem. Soc. 2005; 127(11):4060-4070.

9. Gaur U, Sahoo SK, De TK, Ghosh PC, Mitra A, Ghosh PK. Biodistribution of fluoresceinated dextran using novel nanoparticles evading reticuloendothelial system. Int. J. Pharm. 2000; 202:110. [PubMed: 10915921]

10. Simberg D, Duza T, Park JH, Essler M, Pilch J, Zhang L, Derfus AM, Yang M, Hoffman RM, Bhatia S, Sailor MJ, Ruoslahti E. Biometric amplification of nanoparticle homing to tumors. Proc. Natl. Acad. Sci. 2007; 104:932-936. [PubMed: 17215365]

11. Bonnemain B. Superparamagnetic agents in magnetic resonance imaging: physiological characteristics and clinical applications-a review. J. Drug Target. 1998; 6:167-174. [PubMed: 9888302]

12. Wang YX, Hussain SM, Krestin GP. Superparamagnetic iron oxide contrast agents: physicochemical characteristics and applications in MR imaging. Eur. Radiol. 2001; 11:23192331. [PubMed: 11702180] 


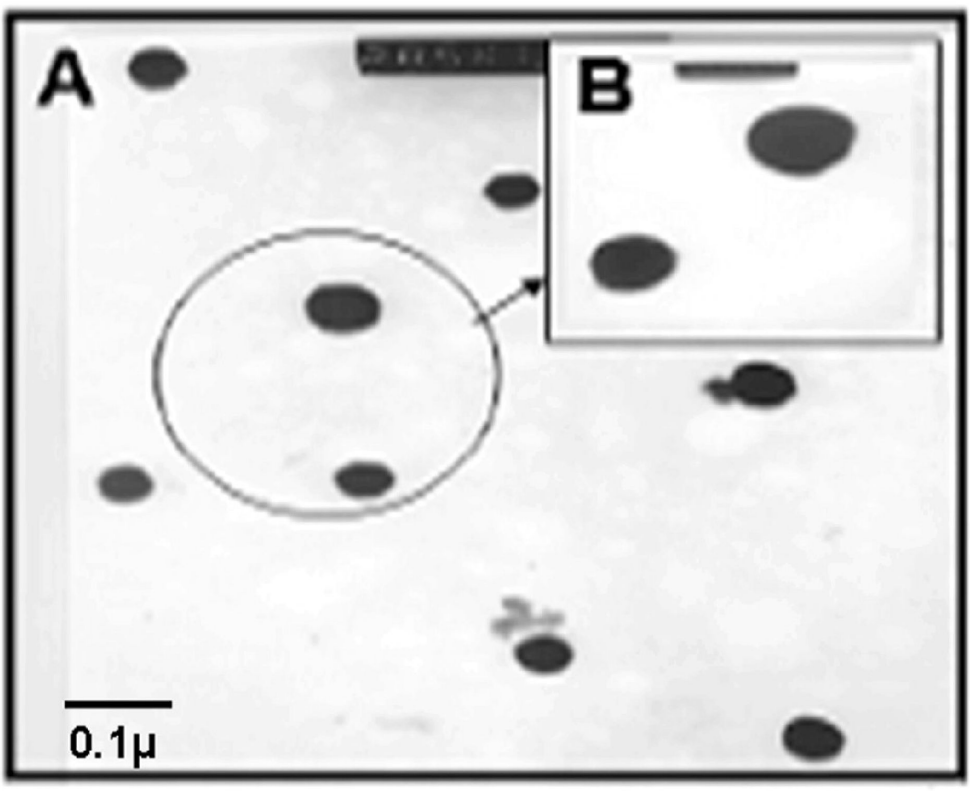

Figure 1.

(A) TEM picture of $\mathrm{Fe}_{2} \mathrm{O}_{3}$ nanoparticles, size $60 \mathrm{~nm}-70 \mathrm{~nm}$ and (B) is the magnification of $\mathrm{Fe}_{2} \mathrm{O}_{3}$ nanoparticles. 


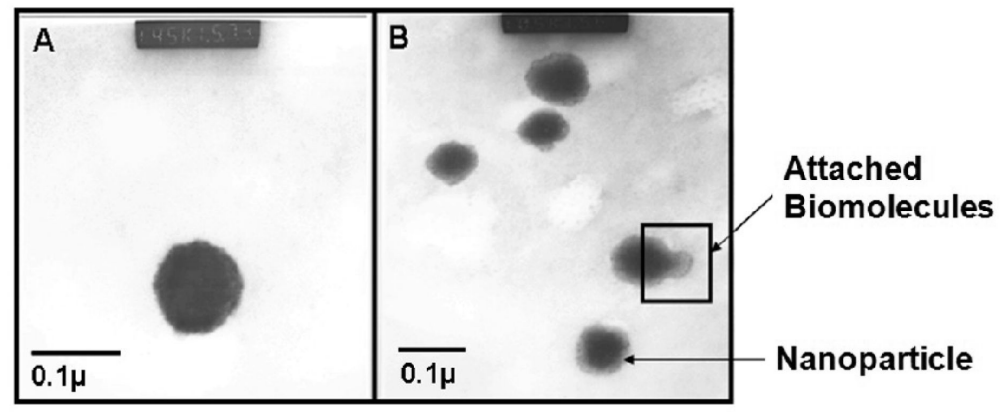

Figure 2.

TEM pictures of (A) $\mathrm{Fe}_{3} \mathrm{O}_{4}$ nanoparticles without modification and (B) after modification with CEA antibodies. 


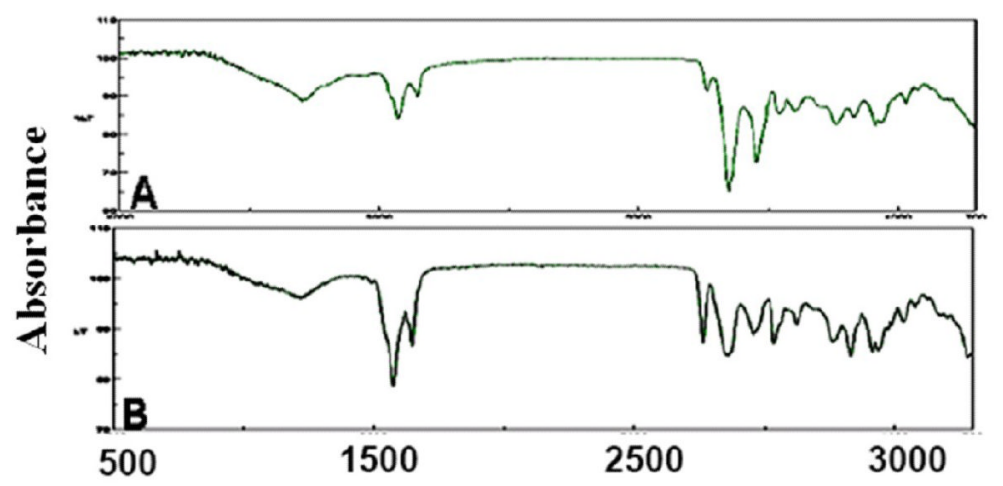

Wavenumber $\mathrm{cm}^{-1}$

Figure 3.

Fourier transform infrared spectroscopy (FTIR) spectra of (A) $\mathrm{Fe}_{2} \mathrm{O}_{3}$ magnetic nanoparticles and (B) $\mathrm{Fe}_{2} \mathrm{O}_{3}$ magnetic nanoparticles coupled with CEA antibodies. The stronger absorption peak in $\mathrm{B}$, confirms the binding of CEA antibodies to the $\mathrm{Fe}_{2} \mathrm{O}_{3}$ magnetic nanoparticles. 
A.

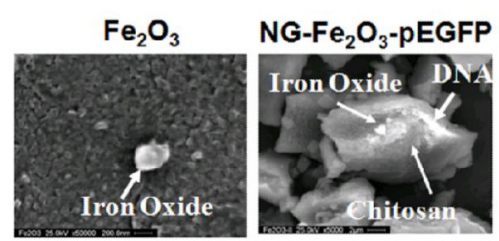

B.

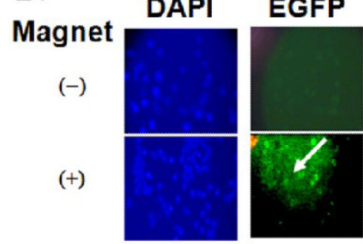

C.

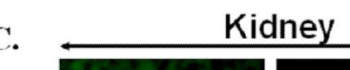

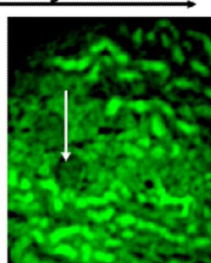

Yes

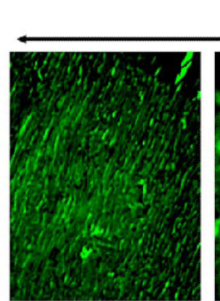

No
Heart

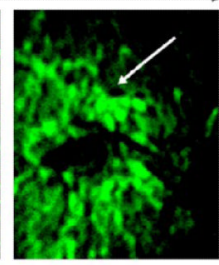

Yes

Figure 4.

(A) is an SEM micrograph of $\mathrm{Fe}_{2} \mathrm{O}_{3}$ alone and in complex with EGFP DNA and chitosan. (B) Localization of the magnetic nanoparticles coupled with EGFP DNA to the thoraces cavity using external magnetic influence. (C) Localization of the magnetic nanoparticles coupled with EGFP DNA to the heart and kidney. The fluorescence microscopic picture shows the expression of EGFP gene at target site where the magnet is placed externally. The expression of EGFP is denoted by the arrows. In contrast, when there is no external magnet, the EGFP gene does not retain at the target site, as evidenced by the absence of the EGFP protein in the heart or kidney. 


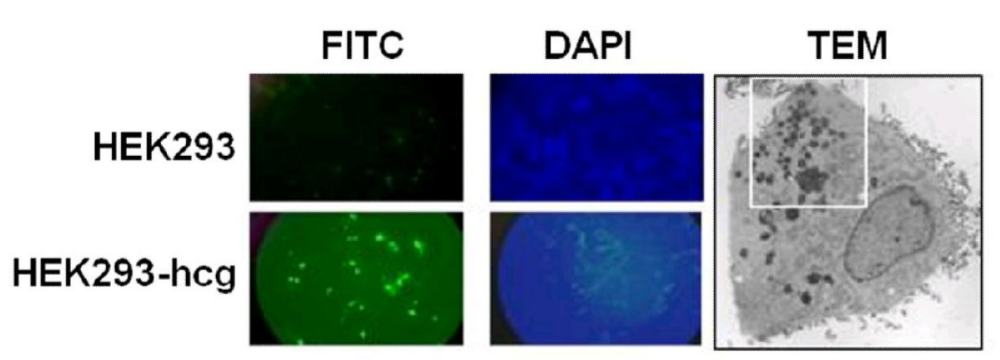

Figure 5.

Fluorescent micrographs of HEK293 and HEK293hcg cells that were transfected with magnetic nanoparticles coupled with ANP peptide. TEM picture of HEK293hcg cell showing the functionalized magnetic nanoparticles inside the cell. 


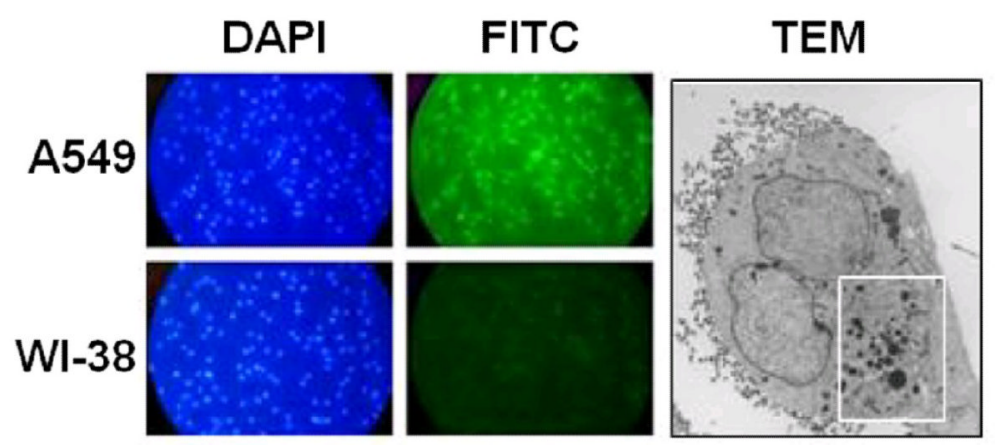

Figure 6.

Fluorescent micrographs of A549 and WI-38 cells that were transfected with magnetic nanoparticles coupled with CEA antibody. TEM picture of A549 cell showing the functionalized magnetic nanoparticles inside the cell. 